http://dx.doi.org/10.21611/qirt.1992.016

\title{
Application of quantitative infrared thermography in the VKI Mach 6 hypersonic wind tunnel
}

\author{
by G. SIMEONIDES, J.P. VERMEULEN and S. ZEMSCH
}

von Karman Institute for Fluid Dynamics, Chaussée de Waterloo 72, B-1640 Rhode St-Genèse, Belgium.

\begin{abstract}
The application of infrared thermography to the measurement of heat transfer over models tested in the VKI H-3 hypersonic wind tunnel at a Mach number of 6 is described. The performance of the technique in quantifying heat transfer rates over aerodynamic surfaces is found to be comparable to that of discrete point gauges, such as thin film surface resistance thermometers and coaxial thermocouples. The merits and limitations of infrared thermography in wind tunnel testing are illustrated by a series of examples representing quantitative heat transfer measurements over regions of hypersonic shock wave boundary layer interactions.
\end{abstract}

\section{Introduction}

The boost in hypersonic aerothermodynamic research and the importance of heat transfer in the design of hypersonic lifting vehicles have led to the development and application of thermographic techniques which may efficiently (in terms of time and cost) provide two-dimensional thermal maps over complex aerodynamic surfaces. Amongst them, infrared thermography has been particularly successful thanks to the commercialization of high performance infrared scanning radiometers (infrared cameras) and (black and white) Digital Image Processing (DIP) systems.

The technique has been applied for quantitative heat transfer measurements in the VKI Mach $6 \mathrm{H}-3$ blowdown wind tunnel [1] and, in the course of the past five years, it has been developed to provide data of comparable quality to those given by discrete point gauges [2-4]. In what follows, a brief description of infrared thermography is given, as this is applied in the VKI hypersonics laboratory, together with a presentation of selected results to illustrate the advantages and limitations of the technique. The selected model configurations are simplified geometric representations of regions of shock wave boundary layer interaction over hypersonic lifting vehicles, such as deflected control surfaces and wing-body junctions, and are illustrated in figure 1.

\section{Measurement chain and data quality}

\subsection{Measurement chain}

An Inframetrics Model 525 infrared camera, equipped with an $\mathrm{HgCdTe}$ detector sensitive in the 8-12 $\mu \mathrm{m}$ wavelength range, has been employed for the measurements. The total field of view with the standard optical setup of this camera is $14^{\circ}$ vertical $\times 18^{\circ}$ horizontal, and the corresponding instantaneous field of view (IFOV) is 2 mrad. With a $3 \times$ afocal telescope lens, used to enhance spatial resolution, the IFOV reduces to $0.7 \mathrm{mrad}$. The typical operational distance of the camera from the measurement surface has been approximately $0.65 \mathrm{~m}$, and the typical dimensions of the model surface $150-200 \mathrm{~mm}$ length and $100 \mathrm{~mm}$ span. A germanium viewing window, transparent in the $8-12 \mu \mathrm{m}$ infrared wavelength range, has been necessarily installed in the tunnel wall since the test chamber of the tunnel is evacuated during an experiment.

The output of the infrared camera is video recorded and the measured surface intensity data are transmitted to the computer through a Data Translation Inc. DIP system for data reduction. The measured intensity distributions are decalibrated into surface temperature distributions by means of a first or second order calibration curve, and the semi-infinite slab principle $[1,5]$ is employed to convert the time evolution of surface temperature into a heat transfer distribution. To satisfy the requirements of the semi-infinite slab heat conduction model, the wind tunnel models have been constructed of plexiglas, except for the forward $25 \mathrm{~mm}$ long leading edge part that 
was constructed of steel. The measurement surfaces have been painted black to enhance the surface emissivity in the infrared domain. Data are finally presented in the form of a heat transfer coefficient as the heat transfer rate into the measurement surface normalized by the freestream density, velocity and specific heat of the test gas, and by a form of driving temperature difference, namely that between the reservoir and model surface temperatures.

More details about the wind tunnel and models, and the measurement chain and the data reduction procedures may be found in $[1,3]$.

\subsection{System calibration and quality of data}

\subsubsection{Thermal resolution}

The temperature-intensity calibration of the entire measurement chain is made in situ to account for the effects of the finite model surface emissivity and the finite transmissivity of the germanium viewing window. An electrically heated and an unheated plate at ambient temperature are used for this purpose. Both plates are painted with the same mat black paint as the wind tunnel models. Their surface temperatures are monitored by thin foil thermocouples and compared to the DIP intensity measured by the infrared measuring system.

Calibration curves are shown in figure 2 for the 20 degree thermal range of the infrared camera (typically employed in the experiments) and for both the standard optical setup and the teleoptic lens. For this small thermal range, the calibration curves show a linear behaviour and are characterized by an uncertainty band of $\pm 2 \%$ [3] of the full temperature scale. This uncertainty value is comparable to the accuracy levels attained with standard thin film gauges of $1.5-2 \%$ [1]. The limiting factor in the uncertainty of the measurement is the thermal resolution of the infrared scanner itself of only 64 intensity levels compared to the 256 intensity levels of the DIP system and the $48 \mathrm{~dB}$ signal-to-noise ratio of the U-matic video recorder employed in the measurements.

It is pointed out, however, that one of the major limitations of the infrared technique is that a single value of sensitivity of the system must be employed over the entire measurement domain. Consequently, in order to avoid saturation of the signal in high heating regions after a given exposure time of the model in the flow, high signal-to-noise ratios must be tolerated for the same model exposure time in low heating regions. This problem may be circumvented by means of either duplicating the experiments employing two different sensitivities (thermal ranges) of the camera or employing two different data reduction times - a short one for high heating regions and a longer one for low heating regions.

\subsubsection{Spatial resolution}

When the objects (or temperature variations) observed by the infrared camera have a size of the same order as or smaller than the area covered by the system IFOV, then the desired intensity distribution is not adequately resolved. The resulting signals are spread in space and reduced intensity amplitudes are measured. A useful rule of thumb is that losses due to limited spatial resolution become important with surface intensity (temperature) variations that occur over distances of less than 5 to 10 times the system IFOV. It is noted that the spatial characteristics of the infrared camera for an operational distance of $0.5 \mathrm{~m}$, namely an IFOV of $1 \mathrm{~mm}(0.35$ $\mathrm{mm}$ with the $3 \times$ telescope) and the corresponding size necessary for temperature variations to remain unaffected by spatial resolution of $5-10 \mathrm{~mm}(1.75-3.5 \mathrm{~mm}$ with the $3 \times$ telescope), are comparable to the typical width and spacing of thin film gauges of $1 \mathrm{~mm}$ and $5 \mathrm{~mm}$ respectively.

The loss in intensity amplitude of the measurement system due to spatial resolution [6] is characterized by its response to a slit temperature jump (Slit Response Function, SRF) illustrated in figure 3 , or by its response to a square wave temperature variation (Modulation Transfer Function, MTF) illustrated in figure 4 for both the standard optical setup and the $3 \times$ telescopic lens. The limiting factor in the spatial resolution of the system is, as with thermal resolution, the infrared camera itself. Specifically, the 157 independent lines with 157 resolvable elements per line provided by the Inframetrics 525 camera are to be contrasted to the $512 \times 512$ pixels of the 
DIP system and the 625 lines with 250-300 points per line provided by the video recorder.

\subsubsection{Time resolution}

The time resolution of the infrared measurement system employed may be defined either by the $25 \mathrm{~Hz}$ video-compatible frame acquisition frequency with a $2: 1$ interlace which gives a \pm 20 ms time variation within each acquisition image (frame), or by the $50 \mathrm{~Hz}$ scanning frequency of the vertical scanning mirror of the camera which gives a $\pm 10 \mathrm{~ms}$ time variation within each non-interlaced image (field). Data reduction herein is based on the latter $50 \mathrm{~Hz}$ non-interlaced fields. Time resolution may be eventually increased to $7866 \mathrm{~Hz}$ by scanning a single line rather than a two-dimensional surface, in which case the sampling time increment for each point on the measurement surface reduces to $0.12 \mathrm{~ms}$.

\section{Results and discussion}

\subsection{Validation of the technique}

The infrared technique has been validated in [2] against heat transfer measurements made over flat plates and flat plate / two-dimensional ramp configurations with thin film surface resistance thermometers. The favourable comparison between the infrared heat transfer data and the predictions of the reference temperature theory $[7,8]$ in attached flow regions has also provided for gaining confidence in the technique $[3,8,9]$. An example is shown in figure 5 , where the results of the two independent measurement techniques are in close agreement with the theoretical predictions.

\subsection{Flat plate/two-dimensional ramp interactions and striation heating}

Infrared thermography has been extensively employed in the study of shock wave boundary layer interactions over two-dimensional compression corners $[8,9]$ to quantify peak heating levels, attained on the ramp downstream of flow reattachment, as well as the significant spanwise heat transfer variations caused by the formation of Görtler-type vortices in the reattachment region.

Typical spanwise variations of the heat transfer coefficient at a station shortly downstream of flow reattachment on the ramp are shown in figure 6 for a ramp deflected by $10^{\circ}$ installed after a $40 \mathrm{~mm}$ long flat plate. Data are shown for two flat plates with significantly different leading edges. In the former case, a clean leading edge with a thickness of $40 \mu \mathrm{m} \pm 20 \mu \mathrm{m}$ has been employed, and the spanwise heat transfer variations are directly compared to the leading edge thickness distribution. In the second case, a thicker more uniform leading edge with a thickness of $98 \mu \mathrm{m}$ $\pm 5 \mu \mathrm{m}$ has been employed, but with the addition of a regular distribution of $200 \mu \mathrm{m}$ thick, 25 $\mathrm{mm}$ long and $10 \mathrm{~mm}$ wide sandpaper strips installed along the leading edge with a spacing of $10 \mathrm{~mm}$. The corresponding infrared image (with two layers of $10 \mathrm{~mm}$ sandpaper strips) and a sublimation photograph at the same test conditions are shown in colour plate $A^{*}$ to illustrate the strongly three-dimensional heat transfer distribution caused by the roughness distribution along the model leading edge.

It is noted that spanwise heat transfer variations in excess of $20 \%$ are found in the clean leading edge case, which in the presence of additional disturbances reach levels close to $\pm 60 \%$. The bands between the minimum and maximum streamwise heat transfer distributions for the tests illustrated in figure 6 are shown in figure 7 . In the former clean leading edge case, significant spanwise variations are found in the vicinity of flow reattachment. Downstream, the amplitude of spanwise variations diminishes and the heat transfer level tends to that predicted by the reference temperature theory for a turbulent boundary layer at the ramp conditions and with its virtual origin at reattachment. In the case of additional disturbances, significant spanwise variations in heat transfer commence again in the close vicinity of reattachment but pertain to the downstream end of the measurement domain. The predicted ramp turbulent heating level is, however, not exceeded at any spanwise location over the ramp similarly to the former clean leading edge case.

* The colour plates of this article 16 are.located on page $V$ of the colour gathering, at the end of the book 
http://dx.doi.org/10.21611/qirt.1992.016

\subsection{Fin-Induced interactions}

Infrared thermography has also been employed in the $\mathrm{H}-3$ wind tunnel to study the flow over flat plate/swept or unswept blunt or sharp fin configurations, with emphasis placed on the expansion side flowfield and the formation of striations in this region [10]. Example infrared thermograms are shown in colour plate $B$ for a blunt fin with its leading edge swept by $75^{\circ}$ at an angle of attack of $40^{\circ}$. The leftmost thermogram shows the entire measurement domain with the standard optical setup, and the second thermogram shows an artificial zoom of the former (with no gain in spatial resolution) over the area covered with the $3 \times$ telescope in the third thermogram.

A contour plot of the heat transfer distribution, corresponding to the leftmost infrared thermogram of colour plate $B$, is shown in the lower part of figure 8 . Heat transfer distributions along the undisturbed flat plate part of the model and along selected hot areas in the interaction region are plotted in the upper part of figure 8 . The former undisturbed distribution remains laminar throughout the measurement domain as illustrated by the comparison to the laminar reference prediction of curve no.1.

In the case of curve no.4, the measured data downstream of the fin interaction are compared to two turbulent theoretical predictions. Curve no.2 corresponds to the pressure level measured shortly downstream of reattachment of 0.7 times the freestream pressure, and curve no. 3 corresponds to the freestream pressure level reached further downstream. These comparisons illustrate that laminar-turbulent transition is promoted by the interaction in the close vicinity of reattachment, and that the subsequent heat transfer rise is well accounted for by the associated increase in pressure. In the case of curve no.5, laminar-turbulent transition is occurring again in the close vicinity of reattachment, but the lack of pressure data along this curve does not allow for a direct comparison to the reference temperature prediction. Qualitatively, however, a higher pressure level than in the freestream (upstream of the interaction) may be expected shortly downstream of reattachment and a subsequent relaxation to the undisturbed freestream pressure level. These trends are consistent with the comparison between curves no. 3 and 5 in figure 8 and their eventual agreement.

\section{Conclusions}

Infrared thermography, as applied for quantitative heat transfer measurements in the Mach $6 \mathrm{H}-3$ wind tunnel of the von Karman Institute, has been described and its utility illustrated by a series of examples. The performance of the technique in terms of thermal, spatial and time resolution, that is similar to that of standard discrete point gauges, has allowed it to become the prime kinetic heating measurement technique in the $\mathrm{H}-3$ facility. Among its advantages lie the rapid turnaround times for the measurement of heat transfer over complex three-dimensional geometries, aided by the availability of comprehensive black and white DIP systems. A major but easily alleviated limitation is the necessity to employ a single value of sensitivity over the entire measurement domain and, for highly curved measurement surfaces, the directional sensitivity of surface emissivity.

\section{REFERENCES}

[1] SIMEONIDES (G.). - The VKI hypersonic wind tunnels and associated measurement techniques. von Karman institute TM 46, November 1990.

[2] SIMEONIDES (G.), VAN LIERDE (P.), VAN DER STICHELE (S.), CAPRIOTTI (D.) and WENDT (J.F.). - Infrared thermography in blowdown and intermittent hypersonic facilities. AIAA J. Thermophysics \& Heat Transfer, 4, No. 2, April 1990, p. 143-148.

[3] SIMEONIDES (G.), VERMEULEN (J.P.), BOERRIGTER (H.L.) and WENDT (J.F.). - Quantitative heat transfer measurements in hypersonic wind tunnels by means of infrared thermography. IEEE Trans. Aerospace and Electronic Systems, 29, No. 3, July 1993. Proc. 14th Int. Congress on Instrumentation in Aerospace Simulation Facilities, October 1991, p. 178-189.

[4] BOERRIGTER (H.L.). - An introduction to the use of infrared thermography in the $\mathrm{H}-3$ wind tunnel. von Karman Institute, IN 90, June 1991. 
[5] SCHULTZ (D.L.) and JONES (T.V.). - Heat transfer measurements in short duration facilities. AGARDograph 165 , February 1973.

[6] GAUSSORGUES (G.). - La thermographie infrarouge. Paris, Lavoisier, 1981.

[7] NEUMANN (R.D.) and HAYES (J.R.). - Introduction to aerodynamic heating analysis of supersonic missiles. "Tactical Missile Aerodynamics", 104, AIAA Progress in Astronautics \& Aeronautics, eds. M.J. Hemsch \& J.N. Nielsen, 1986.

[8] SIMEONIDES (G.). - Hypersonic shock wave boundary layer interactions over compression corners. Ph.D. Thesis, U. Bristol / von Karman Institute, April 1992.

[9] VERMEULEN (J.P.) and SIMEONIDES (G.). - Parametric studies of shock wave boundary layer interactions in two-dimensional compression corners at Mach 6. von Karman Institute TN 181, September 1992.

[10] ZEMSCH (S.), STOPPER (J.) and DEGREZ (G.). - Wing-fuselage interferences in supersonic flow. EUROMECH 289, April 1992.

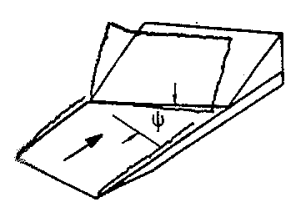

TWO ANO THREE

DIMENSIONAL

COMPRESSION CORNERS

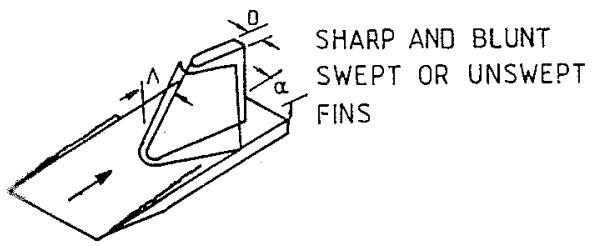

Iig. 1.- Wind tunnel model configurations
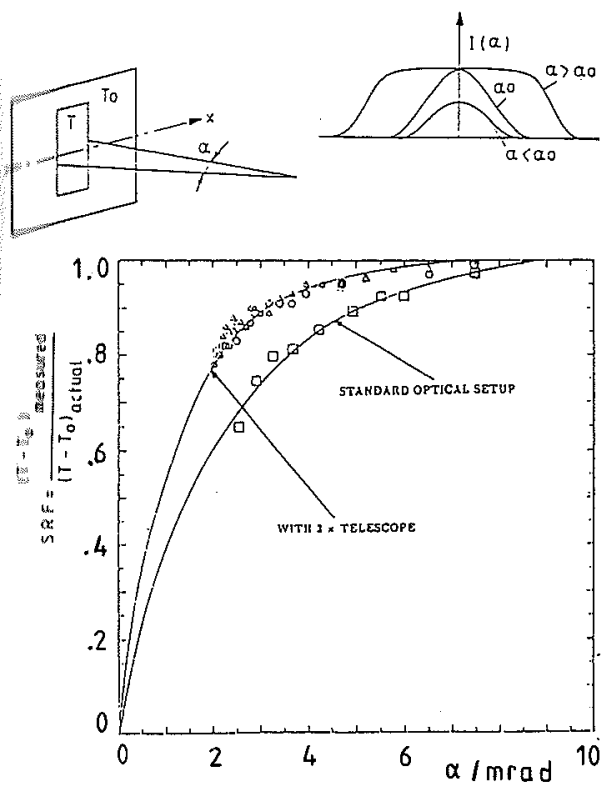

Nig. 3.- Calibrated slit response function

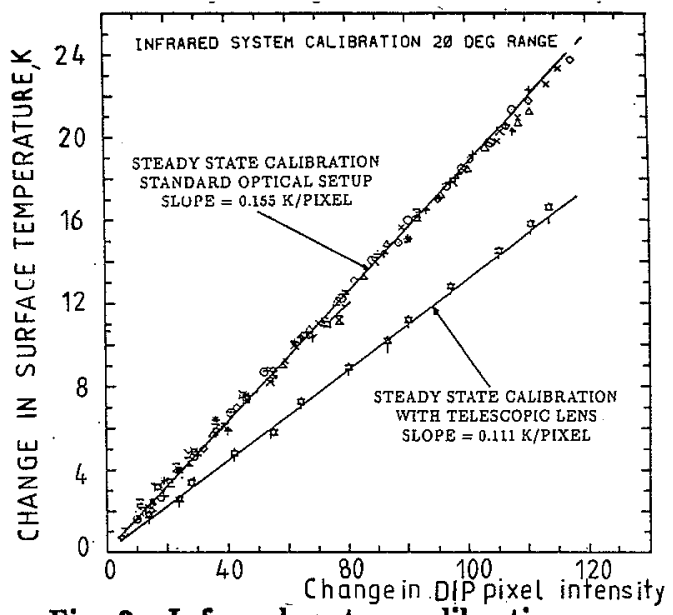

Fig. 2.- Infrared system calibration curves
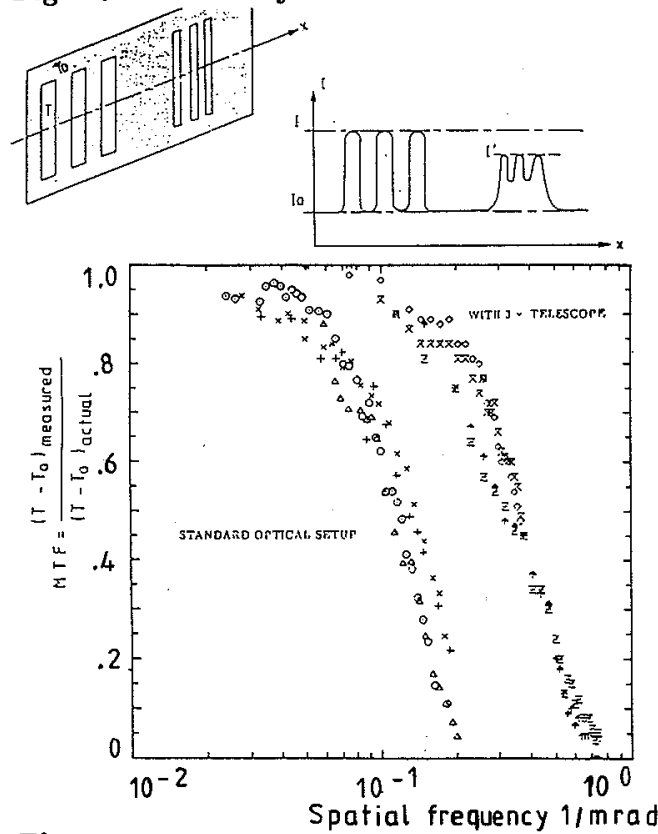

Fig.4.- Calibrated modulation transfer function 


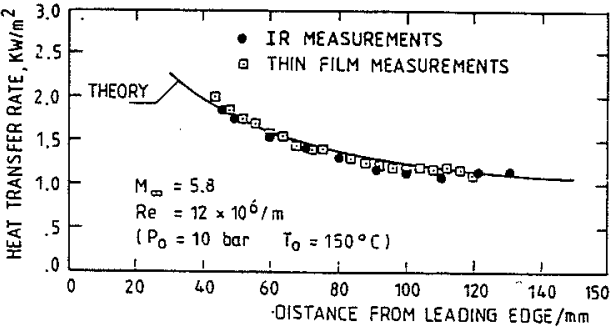

Fig. 5.- Flat plate heat transfer distribution infrared vs thin fllm data vs theory

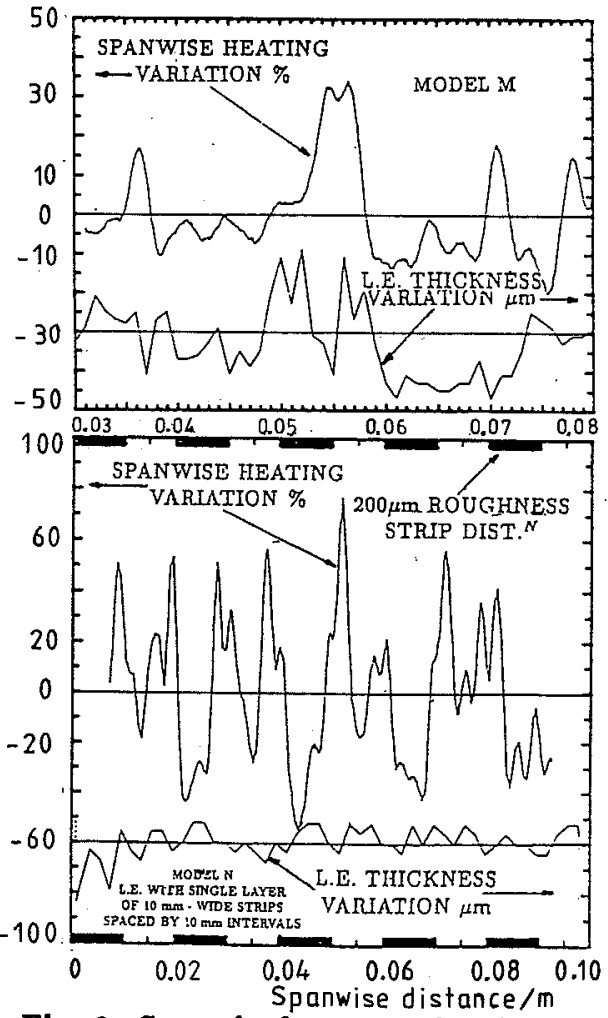

Fig. 6.- Spanwise heat transfer distributions in the vicinity of reattachment on a $10^{\circ}$ ramp
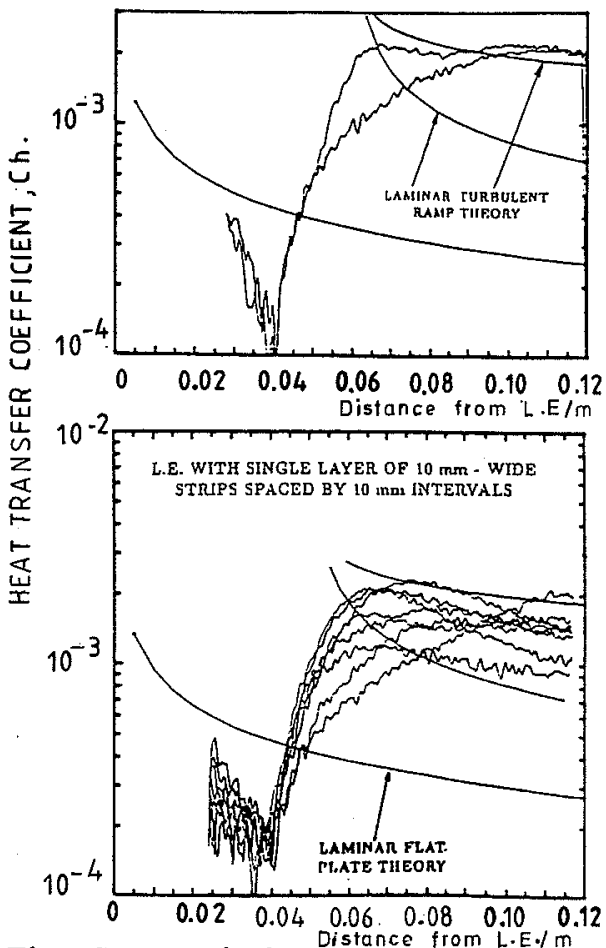

Fig.7-Streamwise heat transfer distributionu along the flat plate $/ 10^{\circ}$ ramp geometry

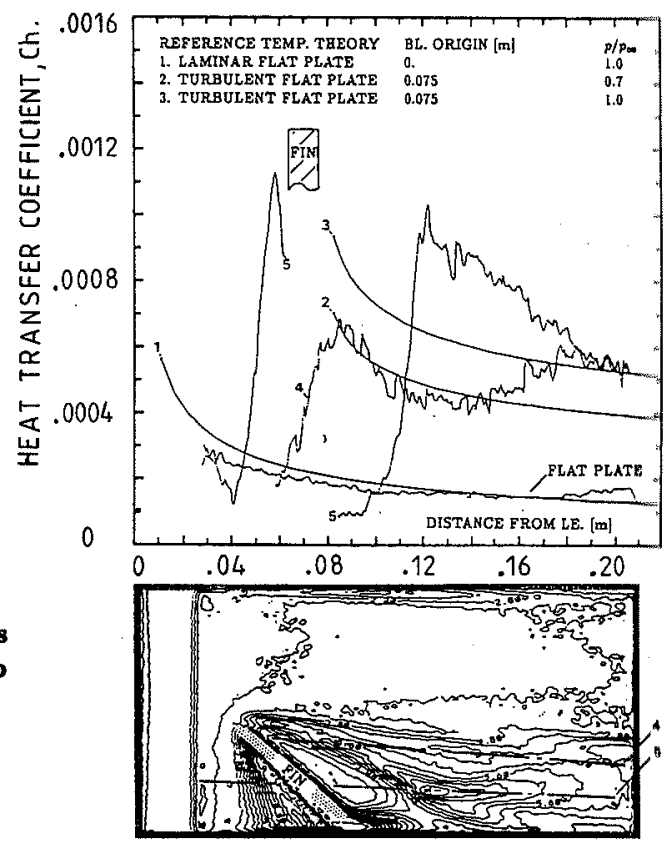

Fig. 8.- Heat transfer distribution over the flat plate/swept fin geometry 